\title{
The Need for Personnel
}

\author{
By GAYLORD W. ANDERSON, M.D., Dr.P.H.
}

Modern public health requires an adequate supply of well-trained personnel, whether the programs be conducted on a local or an international basis. Such a statement is almost axiomatic and should require no defense, yet experience shows that the truth of it is frequently not appreciated by those responsible for the planning and direction of public health. In too many instances there has been a virtual assumption that a reasonably adequate basic training in medicine, engineering, or nursing constitutes adequate background for the vast responsibilities of community health protection. Many of the failures and shortcomings of public health can be attributed to a lack of suitably qualified personnel.

\section{Modern Concept of Public Health}

This failure to appreciate the need for special training stems from the thinking of an earlier era when the prevailing concepts of disease were couched in terms of toxic emanations from decaying filth. Public health of that period was little more than a program of simple cleanliness and hence had no need of specially trained staff. While no one would question the accomplishments of such activities nor would doubt the potential benefits from their extension to many parts of the modern world, our present concepts of disease are not so delightfully simple.

Today we recognize the influence of a vast

Dr. Anderson is director of the School of Public Health, University of Minnesota, and president of the American Public Health Association. He conducted the sessions on professional health education at the Fourth World Health Assembly in Geneva last May. array of physical, biological, social, and economic forces which operate either singly or in combination to exact a high toll of preventable illness and death. Modern public health is the synthesis of the contributions of a great variety of disciplines-a combination of the physical, natural, and social sciences, with a large component of the arts and the humanities. As such, it needs personnel with a sound foundation in their respective fields, supplemented by an understanding of the application of these disciplines to the prevention of disease and a broad appreciation of the interrelationships of the several fields of learning as they impinge upon public health.

A simple example will suffice to illustrate this need. The modern malaria control program is based upon the contributions of the physician, the parasitologist, the entomologist, the chemist, the toxicologist, the pharmacologist, the engineer, the nurse, and the educator. None of them can understand his or her role without some appreciation of the social, economic, political, and climatologic forces that condition the strangle hold that malaria has held on certain areas. Each must understand the relationship of his contribution to the total program for malaria control. Each must further realize that malaria control, like all other programs of public health, is a team operation in which each member must understand his particular function and its relationship to the tasks assigned to his associates. Unless this concept of teamwork is recognized, public health becomes a chaotic jumble of uncoordinated and, at times, conflicting programs, each of which is well-intentioned, but failing of its full potential contribution because it is not properly integrated with other closely related programs.

The training of personnel thus becomes one of the foundation stones of modern public 
health. In the development of its world-wide program, the World Health Organization, following the example of several philanthropic foundations, has therefore very properly given major emphasis to training. A sizable fraction of its very limited budget has been set aside for this purpose. Some of it has been assigned to the betterment of training facilities within various countries. Another large part has been allocated to individual grants whereby selected personnel might be sent to appropriate centers for specialized professional education.

\section{Varied Personnel Needs}

The needs for personnel to be trained must obviously vary greatly from one country to another according to the culture and the problems of the respective nations. In the United States we speak glibly of the separation of curative and preventive services and toy with desired ratios between populations and the numbers of health officers, physicians, nurses, and sanitation personnel. Certainly there is no basis for assuming that such ratios have any meaning outside of our own area. In one country the most urgent need may be for more and better-trained physicians, for in that area there can be no fictitious separation of therapy and prevention. Another nation may have an acute shortage of nurses; in a third the sanitary engineer may be all but unknown, and a fourth may require physicians skilled in some medical specialty.

It would be highly unrealistic to pretend that any predetermined uniform standard might be used to describe the personnel needs of any country. We must recognize as a cardinal principle the fact that the needs of each country must be individually determined and that no agency such as WHO can ever do more than suggest to a nation the direction in which it should proceed in determining its training needs and allocating its training grants.

\section{Personnel Selection}

It would seem axiomatic, however, that in the selection of personnel to receive such grants, preference should be given to those who, upon return to their respective countries, will be in a position to influence the development of sound programs and to contribute to the further training of their fellow countrymen. These persons must be sufficiently advanced in knowledge and experience so that they can profit from the stimulus of new contacts and ideas.

We must not expect, however, that all such students will be sent to one or two foreign countries well-equipped with training institutions. No nation can lay claim to a monopoly in public health knowledge, nor could the schools of any one country pretend to be able to furnish the detailed instruction requisite to meet all the health needs of areas of such different problems, customs, and culture. There is no suggestion that the health programs or procedures developed in one country are necessarily applicable to a distant land.

Those who are sent for foreign study must be persons with a background and understanding which will enable them to separate the basic principles from the operative details and select those ideas which are transferable to, or may be modified to meet, the problems of their respective countries. No greater error could be made either by student or teacher than to assume that the public health program of one nation could ever be transposed in its entirety to any other land.

\section{Regional Training Centers}

It would be equally fallacious to expect that every country, regardless of size, would ever be prepared to maintain full training facilities for all public health personnel. While most countries possess or can look forward to the establishment of schools of medicine, nursing, and engineering, there can be no justification for comparable development of schools of public health.

Rather we must envision regional centers serving nations of comparable culture and language, centers which will provide the basic training needed for those who are entering upon a career in public health. There can be little doubt of the waste of time and money when a person without a background of experience is sent to obtain basic public health training in a country whose language he does not under- 
To be solved ........ Recruitment and Training of Personnel for World Health

- How can the training of additional personnel-particularly health officers, physicians, nurses, and engineers-be further facilitated, and to what extent?

- How far should training needs be met by the development of professional schools in areas where such facilities are now lacking?

- What responsibilities have universities and health departments in the United States for the temporary release of personnel for foreign service?

- What responsibilities has the United States for providing training facilities for foreign students?

- How far, and in what ways, can the limited supply of highly trained professional personnel be supplemented by the preparation of auxiliary workers-medical and nurses aides, midwives, sanitarians? stand. Such a student learns but little and, amidst the linguistic handicaps, finds himself unable to extract from the myriad details those few basic principles which, with suitable modification, he might apply in the position to which he will return. He becomes both confused and discouraged by the details which he recognizes as inapplicable to his country. Regional training centers, even if they lack some of the facilities of large foreign universities, would nonetheless serve a more fundamental need in the training of the great mass of personnel required for the public health programs of the various nations. Foreign study should be reserved for the advanced student.

\section{Broad Training Programs}

In planning for training programs, we must not forget that provision must be made for many types of personnel. I have already stressed the point that an effective public health program depends upon the contributions of persons of a wide diversity of professional backgrounds. If I may criticize our present training programs, I should say that we have given too little attention to nonmedical personnel. There has been too great a tendency on the part of schools of public health to overlook their responsibilities for the training of a coordinated team of workers.

We cannot leave the training of our co-workers to the mercy of professional schools having little or no real interest in public health. The modern school of public health should be a place which receives professionally trained persons from a wide variety of disciplines and adds to their existing knowledge an understanding of the application of their specialities to public health problems and a grasp of the interrelationships existing between the contributions of each profession. The educational isolationism that has characterized so many of our professional schools is as unrealistic as was the political isolationism of an earlier generation.

Equally important in any training program is recognition of the needs of the subprofessional auxiliary worker. It must be quite obvious that the many tasks of public health do not uniformly require professional skills and that many countries will not possess within the foreseeable future an adequate supply of professional persons to perform the duties that one might wish to assign to them. It would be quite unrealistic to sit back and do nothing because of a shortage of persons of desirable professional background.

Many of the duties in environmental sanitation can be assigned to sanitarians lacking engineering background, and many of the instructional duties of the public health nurse may be carried out by a nursing aide, or home visitor. There are many situations in which the limited funds available for public health can be spent to best advantage in the employment of such personnel, provided they have had adequate training for the tasks to which they are to be assigned. 
The problem of subprofessional training is simpler than that of professional education as it requires less time and expense. It should, however, be recognized as an important and fundamental part of a public health training program and not relegated to incompetent hands on the grounds that it is beneath the dignity of an academic institution.

Finally, may I stress the vital importance of the highest academic standards in any public health training program. It would seem unnecessary to point out that, dependent upon the various levels at which training is to be given, the academic standards should be the same as for other professions. Yet the personnel shortages are so great and the demand for personnel so insistent that some have suggested lower standards as a means of attracting persons into public health work. Nothing could be more shortsighted, for the lowering of academic standards would automatically result in a deterioration in the professional quality of work and deter persons of real ability from seeking careers in public health.

What is needed today in all countries is an elevation of professional standards so that public health will attract the highest quality of personnel. Only in this way will we be able to provide personnel of the quality needed to carry on the manifold responsibilities of the program.

\title{
Meeting World Health Problems, 3
}

\section{The Need for Public Understanding and Support}

\author{
By A. W. DENT, LL.D.
}

Public health as the science and practice of preventing disease and infirmity through community efforts has developed slowly over the centuries, beginning first with quarantine laws as the barrier device to protect a well community from the invasion of epidemic disease carried by a sick person. Not very long ago some cities actually prohibited any newcomer from entering the gates until he had lived outside for a prescribed period, to prove that he was not sick nor likely to become sick.

Today such measures are impracticable. A weill man exposed to a virulent form of influenza in New York could, traveling by air,

Dr. Dent is president of Dillard University, New Orleans. He was a member of the United States delegation to the First World Health Assembly in 1948, and is now a member of the steering committee of the National Citizens Committee for the World Health Organization. develop the infectious stage a few days later in India and conceivably continue on through Japan to San Francisco back to New York before being hospitalized, spreading the virus en route.

Moreover, mosquitoes, which carry many diseases, are unable to recognize quarantine. Some years ago the world's worst malaria vector, Anopheles gambiae, was found in Brazil, having been imported from Africa. It was eradicated from Brazil by the Rockefeller Foundation at a cost of millions of dollars. Dr. Raymond Fosdick has suggested that it might be cheaper to eradicate or control mosquitoes in populous areas of Africa than to rely on quarantine measures to prevent their introduction into new areas.

Obviously, public health programs need more than the enforcement of regulations to be successful : an educational effort is as necessary as the provision of safe drinking water or the sanitary protection of the food supply. People 